\title{
Under pressure: a review of normal-tension glaucoma
}

\section{BY DEREK MACDONALD, OD, FAAO}

\section{Introduction}

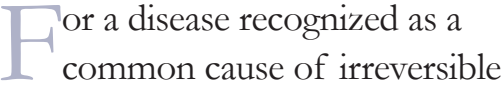
vision loss, a universally agreedupon definition of glaucoma remains elusive. Glaucomatous optic neuropathy (GON) is characterized by a progressive loss of retinal ganglion cells (RGC), resulting in an excavated (cupped) optic nerve head and loss of visual field sensitivity. ${ }^{1}$ Primary open-angle glaucoma (POAG), the most common form of the disease in North America with a prevalence of $2.1 \%$, has been described as "a multifactorial optic neuropathy characterized by acquired loss of retinal ganglion cells and optic nerve atrophy"' This definition has evolved over time, with specific mention of intraocular pressure (IOP) now conspicuously absent. This is at least in part in recognition of the paradox of ocular hypertension (OHT) without accompanying GON, and of GON in the presence of 'normal' IOP; it could be stated that increased IOP is sufficient, although not necessary, for the development of glaucoma. Normal-tension glaucoma (NTG) has been defined as POAG with untreated IOP within the statistically normal range of $15.5+/-2.6 \mathrm{mmHg}$; others specify that high-water IOP cannot exceed $21 \mathrm{mmHg}$, at which point a diagnosis of POAG is established. ${ }^{3}$

Interestingly, while IOP no longer defines POAG, it does define NTG, and remains the single most important, and the only currently modifiable, risk factor in the development of glaucoma. Further, patients with NTG may demonstrate a more aggressive disease if left untreated, but often respond favourably to IOP-lowering treatment. ${ }^{4}$ This has led investigators to suggest that the glaucoma pendulum has swung too far away from IOP, and that the disease may be best defined as the

\section{RÉSUMÉ}

En moyenne, un patient sur trois atteint de neuropathie optique glaucomateuse aura une pression intraoculaire se situant à l'intérieur des limites de la normale et recevra le diagnostic de glaucome à tension normale. Les professionnels des soins oculovisuels (et leurs patients) auront intérêt à bien connaître le diagnostic, le traitement et le pronostic de cette condition et à bien comprendre non seulement les similitudes et les différences avec le glaucome primaire à angle ouvert mais les rôles importants joués par le système nerveux central et l'état vasculaire systémique.

Mots clés : Glaucome à tension normale (GTN), glaucome primaire à angle ouvert (GPAO), hystérèse cornéenne (HC), hémorragie discale (HD), atrophie péripapillaire de la zone bêta (APP $\beta$ ), pression de perfusion oculaire (PPO), dysrégulation vasculaire, pression du liquide céphalorachidien (PLCR), différence de pression à travers la lame criblée, neuroprotection only pressure-dependent optic neuropathy. ${ }^{5}$ Indeed, many recommend that the concept of distinct clinical entities be abandoned in favour of viewing glaucoma as a continuum from primarily IOP-dependent (POAG) to IOP-independent (NTG) disease. ${ }^{6}$ Given that as many as five of every ten patients with glaucoma will present with statistically normal IOP, an understanding of the multifactorial nature of what this review will term NTG is of critical importance to the eye care practitioner.

\section{Epidemiology and Risk Factors}

Even more than POAG, NTG tends to be a disease of the elderly, with a prevalence of $1.6 \%$ in the population over the age of 75 ; up to $30 \%$ of patients with NTG, however, will be under the age of $50 .{ }^{7}$ Upon diagnosis, the rate of progression and response to treatment appear unrelated to age. ${ }^{8}$ There is evidence that NTG is more common, more severe, and more resistant to treatment in females. ${ }^{9}, 10$ There also appears to be an ethnic predilection, as upwards of $90 \%$ of Japanese and Mongolian patients with POAG present with IOP less than $21 \mathrm{mmHg}$; Caucasians, however, tend to manifest more serious disease. ${ }^{11-13}$ A family history of glaucoma is reported by 30 to $40 \%$ of patients with NTG. Investigators have observed that patients with NTG tend to be of lower body weight and body-mass 
index (BMI). ${ }^{14}$ It has been hypothesized that patients with NTG tend to be more health-conscious (in fact, some would suggest healthanxious), and exhibit more proactive health behaviour. Myopic patients may demonstrate progressive GON in the presence of low IOP, and tend to have difficult to interpret, often tilted, optic nerve heads. ${ }^{15,16}$ While a discussion of genetics is beyond the scope of this review, upwards of twenty genes associated with POAG have been identified, and there is evidence that several may be specific for NTG. At least two gene loci are associated with NTG and exfoliative glaucoma; these loci influence transforming growth factor beta (TGF- $\beta$ ), perhaps suggesting a future neuroprotective target. ${ }^{17,18}$

\section{Pathophysiology - Under Pressure}

Although by definition NTG presents with IOP within the statistically normal range, admittedly an arbitrary construct with no pathophysiologic meaning, further reducing pressure tends to slow disease progression, albeit not universally. ${ }^{19}$ Nocturnal IOP elevation, particularly in concert with nocturnal systemic hypotension, is very significant; sleep lab and telemetric studies demonstrate that as many as two out of every three patients exhibit maximal IOP outside regular office hours. ${ }^{20-22}$ In recognition of the impact of corneal biomechanical properties on applanation tonometry (AT), and potentially on ocular integrity itself, these properties have recently received greater attention. Patients with NTG tend to have central corneal thicknesses (CCT) approximately 30 microns below the population mean of 550 microns, leading some to hypothesize that a subset of patients with POAG are misdiagnosed with NTG ${ }^{23,24}$ It has been proposed that the increased prevalence of NTG among some ethnic groups (individuals of Japanese and African descent) may be partly attributable to thin CCT. ${ }^{25,26}$ Interestingly, reduced CCT was more common in patients with NTG and vascular dysregulation than in those without, suggesting more than simply an underestimation of IOP. ${ }^{27}$ While the Ocular Hypertension Treatment Study (OHTS) did lead to fewer patients with OHT and more patients with 'normal' pressures being treated, the association between CCT and glaucoma, specifically whether CCT may be considered a proxy for $\mathrm{ONH}$ biomechanical integrity, remains unclear. ${ }^{28}$ Recently, the role of corneal hysteresis $(\mathrm{CH})$, reflecting the cornea's viscoelastic ability to dampen fluctuations in IOP and reduce optic nerve head $(\mathrm{ONH})$ strain, has received attention as another potentially important biomechanical parameter. ${ }^{29,30}$ While influenced by CCT, lower $\mathrm{CH}$ is consistently and independently associated with an increased risk of GON. ${ }^{31}$ There is evidence that a related parameter, corneal resistance factor (CRF, a measure of ocular rigidity), is similarly reduced in cases of concurrently low but fluctuating IOP - that is, NTG. ${ }^{32}$ Whereas attempts to 'correct' IOP for CCT alone have proven ineffective, 'corneal compensated IOP (IOP ${ }_{c}$ )', encompassing a more global corneal biomechanical analysis, may hold promise: IOP ${ }_{c c}$ was essentially equal to AT in POAG, but significantly higher in NTG. ${ }^{33}$ Whether reduced $\mathrm{CH}$ and $\mathrm{CRF}$ are risk factors for, or a result of glaucoma, and whether they will prove to be better proxies for ONH biomechanical integrity than CCT alone is yet to be determined; further study is necessary. ${ }^{34}$

Reduced ocular perfusion is found in the majority of patients with glaucoma, more so in the presence of NTG than POAG. ${ }^{35}$ Cardiovascular disease, including increased blood viscosity, diabetes,

\section{ABSTRACT}

On average, every third patient with glaucomatous optic neuropathy will present with intraocular pressure within the statistically normal range, manifesting normal-tension glaucoma. Eye care practitioners (and their patients) will benefit from a familiarity with the diagnosis, treatment, and prognosis of this condition, including similarities to, and differences from, primary open-angle glaucoma, and the important roles played by the central nervous system and systemic vascular status.

Key words: normal-tension glaucoma (NTG), primary open-angle glaucoma (POAG), corneal hysteresis $(\mathrm{CH})$, disc hemorrhage $(\mathrm{DH})$, beta-zone peripapillary atrophy ( $\beta P P A)$, ocular perfusion pressure (OPP), vascular dysregulation, cerebrospinal fluid pressure (CSFP), trans-lamina cribrosa pressure differential, neuroprotection 
and both systemic hypertension and hypotension, has been identified as a risk factor for the development of glaucoma, and may be predictive of a poor response to treatment. ${ }^{36,37} \mathrm{In}$ fact, patients tend to show increased risk of glaucoma at both extremes of blood pressure (BP), albeit more so with hypotension, which results in generalized poor perfusion. Hypertension leads to atherosclerosis, damaging endothelial cells and impairing autoregulation, rendering the ONH more susceptible to decreased vascular perfusion, increased IOP, and metabolic demands. ${ }^{38}$ In the Collaborative Normal-Tension Glaucoma Study (CNTGS), patients without cardiovascular disease tended to progress rapidly when untreated, but benefitted from IOP reduction; vasospastic disease was more predictive of progression than occlusive disease. Magnetic resonance imaging (MRI) of the brain has demonstrated vascular insufficiency in patients with NTG, while cardiac studies have reported an increased incidence of silent myocardial infarction. ${ }^{8,38}$ In patients with low IOP who show progressive visual field (VF) and ONH damage, systemic hypotension causing low ocular perfusion pressure (OPP, a surrogate being the difference between brachial BP and IOP) may undermine the benefits of low IOP ${ }^{39-41}$ The risk of GON increases as much as six-fold in the presence of low OPP; a diastolic OPP of less than $55 \mathrm{mmHg}$ has been associated with a doubling of relative risk..$^{42,43}$ A physiologic nocturnal BP dip secondary to reduced sympathetic nervous system activity that coincides with a nocturnal IOP spike can cause a pronounced OPP trough. ${ }^{44,45}$ Patients with nocturnal BP dips of greater than 10 to $15 \%$ demonstrate more significant retinal nerve fiber layer (RNFL) and VF loss. ${ }^{46,47}$ Some patients may experience iatrogenic systemic hypotension secondary to aggressive treatment of systemic hypertension. ${ }^{48,49}$ Indeed, aggressive lowering of BP has been shown to increase $\mathrm{ONH}$ cupping in patients without glaucoma. Significant variations in OPP, like IOP, may be an independent risk factor for GON and VF deterioration within ten degrees of fixation. ${ }^{50-52}$ OPP may be increased by lowering IOP and avoiding overtreatment of systemic hypertension (of course, deliberately elevating BP increases comorbidities), and its variability reduced by smoothing IOP spikes and BP troughs. ${ }^{53}$

Patients with NTG often have histories of tinnitus, migraine headache, and Raynaud's phenomenon, all manifestations of primary vasospastic vascular dysregulation, an imbalance between autoregulatory vasoconstrictor and vasodilator stimuli. ${ }^{4,14}$ Patients with migraine, especially women, seem particularly predisposed to rapid $(2.6 \times)$ progression of NTG, and lowering IOP in women with migraine may be less protective than in those without. ${ }^{8,12,54}$ Vasospastic disease is more common in women, particularly post-menopause, and in patients of Japanese descent, two populations known to be at higher risk of NTG. Hemorrhaging within the fingernail capillary bed, an accepted sign of vascular dysregulation, is statistically more common in patients with glaucoma, particularly in those with a history of disc hemorrhage, and may be a helpful ancillary indication of vascular insufficiency. ${ }^{55}$ Reduced arterial and peripapillary retinal capillary blood flow has been demonstrated in patients with NTG; many of these patients exhibit vasospastic tendencies and asymmetric VF loss that correlates to interocular asymmetries in blood flow and velocity. ${ }^{56,57}$ Episodic vasospasm and rebound hyperperfusion can lead to local inflammation and oxidative damage. ${ }^{35}$ Some patients with presumed GON and statistically normal IOP will have a history of hemodynamic crisis (sudden and severe systemic hypotension); such patients tend to show minimal if any progression over time. ${ }^{36,37}$ In fact, in an early study, Drance noted that nearly $90 \%$ of patients with NTG had experienced transient or sustained systemic hypoperfusion. ${ }^{39}$

While eye care practitioners routinely measure the trans-corneal pressure differential (the difference between IOP and atmospheric pressure), what truly influences the $\mathrm{ONH}$ through disruption of RGC axoplasmic flow is the translamina cribrosa pressure differential (the difference between IOP and orbital cerebrospinal fluid pressure [CSFP])..$^{58,59}$ The elevated translamina cribrosa pressure differential of POAG caused by high IOP may be mimicked in NTG by a low CSFP within the optic nerve subarachnoid space (ON SAS).${ }^{60}$ CSFP 
is lower in patients with NTG than in patients with POAG; both groups exhibit lower CSFP than controls (the average being between 5 and $15 \mathrm{mmHg}$ ), who, in turn, exhibit lower CSFP than patients with OHT, ${ }^{61,62}$ The inter-group CSFP differences appear similar to the inter-group IOP differences observed in other studies. ${ }^{63}$ Low CSFP and high trans-lamina cribrosa pressure differential are both positively correlated with GON and glaucomatous VF loss. ${ }^{64}$ The thinning of the lamina known to occur in GON may exacerbate the trans-laminar pressure differential. Given that pulsatile mechanical stress is more damaging than steady, the role of CSFP fluctuation, akin to IOP fluctuation, is also receiving attention. ${ }^{65}$ In patients with NTG, the density of CSF in the ON SAS is significantly lower than intracranial CSF; this impairs fluid exchange and leads to relative CSF stagnation within the ON SAS, with potentially detrimental impact upon RGC axons. ${ }^{66}$ All three pressures (IOP, OPP, and CSFP) are independent yet interrelated, and may be simultaneously influenced by an as yet undetermined systemic mechanism. ${ }^{67}$ Indeed, one cannot discount the possibility that GON and VF loss attributed to low OPP is actually secondary to low CSFP, as the latter is often found in the presence of systemic hypotension. Neuroimaging has demonstrated a narrower $\mathrm{ON}$ SAS width in patients with NTG, suggesting lower CSFP in that space. ${ }^{68}$ Given that direct CSFP measurement through lumbar puncture (LP) is invasive and not without risk, such a surrogate noninvasive means of assessment would certainly be of value.

\section{Structural Change}

Some investigators feel that NTG exhibits an extreme amount of ONH cupping, typified by a pale, gently sloping, moth-eaten appearance, with broad thinning of the inferior temporal aspect of the neuroretinal rim (NRR) ${ }^{69}$ Others suggest that the disc changes in NTG represent localized areas of nonperfusion (a focal ischemic glaucoma), preceding or coinciding with adjacent wedge or slit RNFL loss that results in initial severe VF loss that is very close to fixation. ${ }^{70}$ This type of damage appears more common in female patients with a history of systemic vasospasm and migraine. ${ }^{71}$ Subsequent confocal scanning laser ophthalmoscopic (SLO) studies, however, found no significant differences in optic disc topography in cases of POAG and NTG. ${ }^{72}$ The rate of progressive $\mathrm{ONH}$ damage may be greater in patients with NTG than in those with POAG, particularly in patients with already-advanced GON, where lowering IOP may be of marginal benefit. $^{73}$

First described by Bjerrum over a century ago, rising to prominence through the work of Drance some sixty years later, the etiology of disc hemorrhages (DH) remains unclear. Rather than arguing cause versus effect (primary infarction versus secondary degeneration), a mixed-mechanism theory is gaining traction. ${ }^{7477}$ These small pre-laminar radial flame- or splinter-shaped hemorrhages occur most commonly at the inferior temporal aspect of the $\mathrm{ONH}$, adjacent to areas of focal
NRR thinning and RNFL loss, and within two clock hours of areas of beta-zone peripapillary atrophy. ${ }^{78-82}$ They are two- to five-fold more common in patients with NTG than in those with POAG or OHT, or without glaucoma. Indeed, 15 to $42 \%$ of patients with NTG demonstrate $\mathrm{DH}$ at baseline or follow-up, versus 7 to $37 \%$ of patients with POAG, $8 \%$ of those with OHT, and only 0.2 to $0.5 \%$ of the non-glaucomatous population. ${ }^{83-88}$ $\mathrm{DH}$ are found frequently in older patients with systemic hypertension, in patients with vasospastic disease, and in women with a history of migraine. ${ }^{89,90}$ They are more common in the presence of IOP instability, and relatively rare in patients with secondary OAG, who typically present with significant IOP elevation. In the Early Manifest Glaucoma Trial (EMGT), over half the participants demonstrated $\mathrm{DH}$ at least once over an average of eight years; most will be found within the first three to five years of diagnosis. ${ }^{91}$ However, given that the prevalence of glaucoma is 2 to $4 \%$, up to $70 \%$ of isolated $\mathrm{DH}$ will be found in patients not (yet) diagnosed with the disease. ${ }^{83} \mathrm{DH}$ are best detected through photography: being transient and subtle, they are overlooked during clinical exam as often as $84 \%$ of the time. Concurrent disease processes, including posterior vitreous detachment, diabetes, or venous occlusion, must be considered in the differential diagnosis.

DH have long been considered a strong and independent risk factor 
for progressive GON, increasing the hazard rate by a factor of four to six, more so in patients with NTG than POAG, particularly in elderly patients with pre-existing VF loss. ${ }^{92-98}$ In patients with OHT, DH were strong indicators of future conversion to POAG, and were up to five times more common following conversion. ${ }^{94}$ In the CNTGS, $\mathrm{DH}$ was considered a reason to initiate or augment therapy, and was a strong predictor of more rapid progression $(2.7 \times)$ of untreated NTG. ${ }^{99}$ Up to two-thirds of VF and three-quarters of $\mathrm{ONH}$ show progressive change following $\mathrm{DH}$; VF loss may occur at two to eight times the rate, particularly when $\mathrm{DH}$ are inferior temporal and/or multiple..$^{74,77,81,90,94,98}$ Eyes with DH were up to fourteen times more likely to have a worsening of RNFL status within one year. RNFL, NRR, and VF loss can also precede DH by weeks or months; retrospective evaluation has indicated that all eyes developing DH show evidence of preexisting NRR notching. ${ }^{100}$ Eyes with enlarging RNFL defects are four times more likely to demonstrate $\mathrm{DH}$, with $80 \%$ occurring at the border between unhealthy and healthy RNFL, suggesting that this is the most active anatomical site of glaucoma progression. Such RNFL defects enlarge toward the fovea nearly $90 \%$ of the time, causing more central VF change. In light of these relationships, some investigators now consider $\mathrm{DH}$ a sign of, rather than a risk factor for, progression. ${ }^{101} \mathrm{DH}$ become less common in end-stage glaucoma, and then are found nasally, adjacent to the only remaining viable NRR and peripapillary vasculature..$^{90,95}$ Once DH is detected, careful documentation and vigilant follow-up is critical; many investigators suggest every few months, given that the average duration of $\mathrm{DH}$ is eight to ten weeks. Recurrent bleeds, often within two years and two clock hours of the initial $\mathrm{DH}$, are found in up to $73 \%$ of patients with NTG; eyes that re-bleed tend to have a significantly lower IOP than eyes with isolated $\mathrm{DH} .{ }^{76,77,87} \mathrm{~A}$ number of studies suggest that patients with recurrent $\mathrm{DH}$ have a higher probability of progressive GON, RNFL loss, and more rapid rates of VF deterioration..$^{102,103}$ As a rule, patients with $\mathrm{DH}$ do not respond as well to treatment as those without. ${ }^{12}$ In fact, moderate IOP lowering may not alter the rate of $\mathrm{DH}$, indicating a less IOP-dependent form of glaucoma requiring more aggressive pressure reduction even in the presence of what would otherwise be considered well-controlled IOP. ${ }^{104}$

Beta-zone peripapillary atrophy ( $\beta P P A)$ is an absence of RPE and thinning of Bruch's membrane and the choriocapillaris immediately adjacent to the $\mathrm{ONH}$; alpha-zone PPA is pigment irregularity just peripheral to the beta-zone when the latter is present. From a semantic perspective, some argue that the term parapapillary is more correct that peripapillary, as the atrophy may not completely encircle the $\mathrm{ONH}$. While present in 15 to $20 \%$ of normal eyes, $\beta$ PPA has been noted to be larger and more frequent in eyes with glaucoma, and is considered an independent, location-specific, and severity-dependent risk factor for the progression of GON. ${ }^{105-108}$ Many believe $\beta P P A$ to be more common in NTG, particularly in younger patients with moderate to severe disease. ${ }^{109-111}$ Other investigators feel that $\beta$ PPA in NTG does not differ from that in POAG, but still helps differentiate NTG from non-glaucomatous optic neuropathy. ${ }^{112}$ Nasal $\beta P P A$ is present in only 1 to $9 \%$ of normal eyes, but 15 to $71 \%$ of glaucomatous eyes; this may also aid in differential diagnosis. ${ }^{13-115}$ Assessing $\beta$ PPA stability may be particularly valuable in the evaluation of small $\mathrm{ONH}$ in which intrapapillary glaucomatous damage can be more difficult to detect. ${ }^{116}$ Conversely, $\beta$ PPA may be less helpful in the evaluation of oblique or highly myopic ONH and in patients of Asian ethnicity, where peripapillary alterations are more prevalent to begin with; ironically, patients with NTG are commonly Asian and/or myopic. ${ }^{117} \beta \mathrm{PPA}$ is often found adjacent to an area of focal NRR loss and/or DH, and large areas of $\beta$ PPA are predictive of future DH. Interestingly, $\beta$ PPA and $\mathrm{DH}$ are associated even in the absence of glaucoma, suggesting a shared etiology of local vascular insufficiency and breakdown of the blood-retina barrier. ${ }^{115}$ Some hypothesize that a disturbance of ONH perfusion secondary to $\beta P P A$ may result in sectoral ischemia, or that leakage of vasoactive substances through compromised peripapillary vessels can damage the RNFL in the face of normal IOP. ${ }^{109}$ In these cases, $\beta$ PPA is felt to be a 
risk factor for, rather than a sequelae of, glaucoma. That being said, $\beta P P A$ is not necessarily static; progression can be seen over time, three to five times more commonly in patients with glaucoma, associated with increasing GON and VF loss. ${ }^{110}$ The presence and enlargement of $\beta P P A$ shows significant correlation with RNFL thickness and rate of thinning (particularly in the inferior quadrant), cup/disc ratio, mean VF loss, and NRR area. ${ }^{114} \beta P P A$ shows a strong correlation with VF defects within five degrees of fixation known to be more common in NTG. Both the absolute scotoma of $\beta P P A$ and the relative scotoma of alpha-zone PPA will cause an enlarged blind spot. $\beta$ PPA can be detected and monitored qualitatively through ophthalmoscopy and photography, quantitatively through imaging techniques including SLO and optical coherence tomography (OCT).

\section{Functional Change}

As already noted, as many as twothirds of cases of NTG present with initial VF defects that threaten fixation; these are strong predictors of future VF deterioration and visual acuity loss. ${ }^{118} \mathrm{VF}$ defects that threaten fixation are best monitored with both 24- or 30-degree and 10-degree testing strategies. Significant VF deterioration appears to occur in one-sixth to one-third of patients with treated NTG. ${ }^{93}$ That being said, recall that the CNTGS showed that over half the patients with untreated NTG manifest no discernible deterioration over five to seven years. While conventional wisdom holds that most cases progress slowly, there is significant variability in rates of progression, even more so than in POAG: a ten-fold range from 0.2 to $2.0 \mathrm{~dB}$ per year. ${ }^{99}$ More VF loss is seen in NTG with higher IOP, but IOP variability over both short- and long-term appears to be an important predictor of, and perhaps independent risk factor for, glaucomatous VF progression, particularly in cases of low IOP. ${ }^{119}$ The challenge, in both NTG and POAG, is to identify those at risk of rapid progression, and initiate early and aggressive treatment. Particular attention must be paid to localized VF progression, which has been proven to be a strong predictor of future $\mathrm{DH}$, and focal GON. ${ }^{74}$ It has long been reported that thinning of the RNFL, documented through both qualitative and quantitative means, is an early sign of GON, often preceding VF loss. ${ }^{120-122}$ Spectral domain OCT (SD OCT) has indicated that RNFL thinning is most significant at the superior and inferior temporal aspects of the $\mathrm{ONH}$, and correlates strongly with VF deterioration. ${ }^{123}$ It has been proposed that loss of 17 to $20 \%$ of age-matched average RNFL thickness, to a level of 70 to 75 microns, is the 'tipping point' for structural change, whereas as many as half the RGC may need to be lost to manifest functional (VF) change. ${ }^{124,125}$ Given that RNFL thickness assessed through OCT demonstrates a floor effect at approximately 50 microns, it may be best to monitor early GON through structural analysis, but advanced GON through functional measures. ${ }^{126}$ Ideally, a combined index of structure and function would allow better detection, prediction, and follow-up at any stage of the disease continuum than either parameter in isolation. ${ }^{127}$

\section{Management}

Given that IOP remains important in the pathogenesis of NTG, the use of topical anti-glaucoma drugs remains the mainstay of treatment. ${ }^{128}$ The CNTGS demonstrated that lowering IOP by $30 \%$ from baseline, to an average of $11 \mathrm{mmHg}$, reduced the risk of progression nearly three-fold. ${ }^{19}$ That being said, $65 \%$ of untreated eyes showed no progression over five years of follow-up, while up to $20 \%$ of treated eyes did. ${ }^{8}$ The EMGT, a study in which over $50 \%$ of the cohort had NTG, indicated that reducing IOP halved the risk of glaucomatous damage, most significantly in the face of alreadylow pressures. ${ }^{129,130}$ The conclusion that each $1 \mathrm{mmHg}$ IOP reduction reduced the risk of glaucoma damage by $10 \%$ emphasized the importance of vigilant monitoring, and that 'last millimeter of mercury of effect' ${ }^{107}$ This aggressiveness must be tempered, however, by the realization that glaucoma treatment is likely to continue for the duration of the patient's life; the side effects of medicine and surgery on quality of life must be carefully considered. ${ }^{131-134}$ Lowering peak and mean IOP and blunting IOP fluctuation decreases the risk and rate of glaucomatous VF loss. ${ }^{135}$ While dealing with an admittedly different population, the Advanced Glaucoma 
Intervention Study (AGIS) indicated that patients with IOP consistently below $18 \mathrm{mmHg}$ demonstrated little if any VF progression over six years; even occasional elevations above $18 \mathrm{mmHg}$ resulted in more VF loss. ${ }^{135}$ Strict adherence to an individualized and appropriate target IOP appears to result in better VF preservation. ${ }^{132}$ Particularly with NTG, clinicians must realize that in-office IOP assessment is but a moment in time, and that structural and functional damage may occur exponentially with undetected IOP spikes. This makes the goals of lowering mean and peak IOP, and smoothing short- and long-term fluctuations, equally critical. In the presence of extreme GON, the disease may become essentially pressure-independent, emphasizing the importance of early and effective intervention.

A review of clinical trials indicates that latanoprost, bimatoprost, timolol, and brimonidine are effective in reducing IOP in patients with NTG: latanoprost seems most effective in reducing trough IOP and smoothing the diurnal curve, while brimonidine is most effective in reducing peak IOP, but least effective at trough. ${ }^{136}$ The World Glaucoma Association recognizes topical carbonic anhydrase inhibitors (CAI) as having a beneficial effect on $\mathrm{ONH}$ perfusion through increasing blood flow velocity in the short posterior ciliary arteries (SPCA); prostaglandin analogs (PA) appear to be hemodynamically neutral. ${ }^{137} \mathrm{PA}$ and CAI lower both diurnal and nocturnal IOP, whereas beta-blockers are ineffective during the nocturnal period. Among the beta-blockers, betaxolol may lower vascular resistance more than timolol, leading to better VF preservation despite higher treated IOP. That being said, should treatment of NTG be initiated, an aggressively low target IOP (approaching episcleral venous pressure of approximately $10 \mathrm{mmHg}$ ) may be preferable; this target may require multiple medications or the consideration of surgery. ${ }^{19}$ In addition to traditional topical management, it has been suggested that systemic calcium channel blockers may be protective in cases of NTG through reduction of vasospasm; others argue against their use due to the potential for nocturnal systemic hypotension and reduced OPP. ${ }^{15,44,73,104}$ Systemic CAI may concurrently lower both IOP and CSFP, leaving the trans-lamina cribrosa pressure differential unchanged, providing little benefit in the management of chronic glaucoma. ${ }^{61}$

As an adjunct, moderate aerobic exercise may be beneficial in both stabilizing the cardiovascular system and reducing IOP. ${ }^{138}$

Neuroprotection is defined as a therapeutic paradigm for slowing or preventing death of neurons (in the case of glaucoma, RGC and their axons) in order to maintain their physiologic function. ${ }^{139}$ Whether secondary to excitotoxic neurotransmitters (glutamate), ischemic/ oxidative injury and subsequent reperfusion inflammation, blockage of growth factors/neurotrophins, mitochondrial dysfunction, or some other mechanism, apoptosis (programmed cell death) may continue independent of the level of IOP. ${ }^{140}$ Glaucomatous damage is not limited to the $\mathrm{ONH}$; alterations in the visual pathway behind the globe (including lateral geniculate nucleus and visual cortex) have been noted in the absence of detectable RGC loss. ${ }^{141}$ Given that current treatments are limited to IOP-lowering, yet some patients with glaucoma continue to progress despite low pressures, a treatment that is independent of IOP is certainly enticing. Some current glaucoma medications appear to have neuroprotective activity: in the Low-Pressure Glaucoma Treatment Study (LoGTS), brimonidine demonstrated a beneficial effect on VF preservation independent of IOP-lowering; as previously noted, dorzolamide has been proven to increase OPP. ${ }^{49,137}$ Memantine and bis(7)-tacrine (glutamate modifiers used in the treatment of Alzheimer's disease, a disease that may share some basic mechanisms of cell death with glaucoma) are among a growing number of systemic agents being studied. ${ }^{142,143}$

In situations where there are atypical clinical findings (age less than 50, visual acuity less than $20 / 40$, ONH pallor, vertically aligned/neurologic VF defects, lack of correlation between structural and functional change, and/or progression at very low IOP) neuroimaging of patients with NTG to rule out compressive lesions of the optic nerve has been suggested. ${ }^{16}$ 


\section{Conclusion}

Normal-tension glaucoma is an increasingly common, and certainly challenging, clinical presentation. The challenge begins with differential diagnosis, and continues through follow-up. Practitioners must gather, integrate, and interpret a myriad of data: ophthalmic and systemic, past and present. Given that many untreated patients show little progression over time, careful observation prior to initiating therapy is certainly prudent. That being said, it may be wise to consider more aggressive treatment of NTG in patients with multiple risk factors - for example, a young female with a history of migraine presenting with a disc hemorrhage. While the mainstay of contemporary management remains topical IOP-lowering, the recognition of ocular perfusion and cerebrospinal fluid pressure as important contributing factors may lead to their modification becoming part of the treatment paradigm.

\section{References}

1. Wax MB, et al. Clinical and ocular histopathological findings in a patient with normal-pressure glaucoma. Arch Ophthalmol 1998;116:993-1001.

2. Krupin T. Special considerations in low-tension glaucoma. Can J Ophthalmol 2007;42:414-7.

3. Park SC, et al. Risk factors for normal-tension glaucoma among subgroups of patients. Arch Ophthalmol 2009;127:1275-83.

4. Nicolela MT. Clinical clues of vascular dysregulation and its association with glaucoma. Can J Ophthalmol 2008;43:337-41.

5. Lichter PR. Expectations from clinical trials. Arch Ophthalmol 2002;120:1371-2.
6. Mroczkowska S, et al. Primary openangle glaucoma vs normal-tension glaucoma. Arch Ophthalmol $2012 \mathrm{Sep}$ 10:1-8;doi:10.1001/2013 (epub ahead of print).

7. Kamal D, Hitchings R. Normal tension glaucoma - a practical approach. $\mathrm{BrJ}$ Ophthalmol 1998;82:835-40.

8. Drance S, et al. Risk factors for progression of visual field abnormalities in normal-tension glaucoma. Am J Ophthalmol 2001;131:699-708.

9. Krupin T, et al. The Low-Pressure Glaucoma Treatment Study (LoGTS). Ophthalmol 2005;112:376-85.

10. Membrey WL, et al. Comparison of visual field progression in patients with normal pressure glaucoma between eyes with and without visual field loss that threatens fixation. $\mathrm{Br} \mathrm{J} \mathrm{Ophthalmol}$ 2000;84:1154-8.

11. Shields MB. Normal-tension glaucoma: is it different from primary openangle glaucoma? Curr Opin Ophthalmol 2008;19:85-8.

12. Anderson DR, et al. Factors that predict the benefit of lowering intraocular pressure in normal tension glaucoma. Am J Ophthalmol 2003;136:820-9.

13. Araie M, et al. Risk factors for progression of normal-tension glaucoma under $\beta$-blocker monotherapy. Acta Ophthalmol 2012;90:337-43.

14. Asrani S, et al. Clinical profiles of primary open angle glaucoma versus normal tension glaucoma patients: a pilot study. Curr Eye Research 2011;35:429-35.

15. Anderson DR, et al. Normal-tension glaucoma. J Glaucoma 2003;12:164-6.

16. Mackenzie PJ, Mikelberg FS, Evaluating optic nerve damage: pearls and pitfalls. Open Ophthalmol J 2009;3:54-8.

17. Gibson J, et al. Genome-wide association of primary open angle glaucoma risk and quantitative traits. Mol Vis 2012;18:1083-92.

18. Wiggs JL, et al. Common variants at 9 p21 and 8 q22 are associated with increased susceptibility to optic nerve degeneration in glaucoma. PLoS Genetics 2008;8:e1002654.

19. Palmberg P. Risk factors for glaucoma progression. Arch Opthalmol 2001;119:897-8.

20. Bagga $\mathrm{H}$, et al. Intraocular pressure measurements throughout the $24 \mathrm{~h}$. Curr Opin Ophthalmol 2009;20:79-83.

21. Lee YR, et al. Circadian (24-hour) pattern of intraocular pressure and visual field damage in eyes with normal-tension glaucoma. Invest Ophthalmol

Vis Sci 2012;53:881-7.

22. Mansouri K, et al. Continuous 24 hour monitoring of intraocular pressure patterns with a contact lens sensor. Arch Ophthalmol 2012 Aug 13;doi10.1001/ archophthalmol.2012.2280:1-6.

23. Doyle A, et al. Central corneal thickness and vascular risk factors in normal tension glaucoma. Acta Ophthalmol Scand 2005;83:191-5.

24. Gordon MO, et al. The Ocular Hypertension Treatment Study. Arch Ophthalmol 2002;120:714-20.

25. Wadhwa SD, Higginbotham EJ. Ethnic differences in glaucoma: prevalence, management, and outcome. Curr Opin Ophthalmol 2005;16:101-6.

26. Kosoko-Lasaki O, et al. Race, ethnicity and prevalence of primary open-angle glaucoma. J Nat Med Assoc 2006;98: 1626-9.

27. Xu L, et al. Central corneal thickness and optic disc hemorrhages: The Beijing Eye Study. Arch Ophthalmol 2008;126:435-6.

28. Robin AL, et al. The Ocular Hypertension Treatment Study. Arch Ophthalmol 2004;122:376-9.

29. Pensyl D, et al. Combining corneal hysteresis with central corneal thickness and intraocular pressure for glaucoma risk assessment. Eye 2012 Aug 10:18;doi:10.1038/eye.2012.164 (epub ahead of print).

30. Brown KE, Congdon HG. Corneal structure and biomechanics: impact on the diagnosis and management of glaucoma. Curr Opin Ophthalmol 2006;17:338-43. 
RIGHTEYE

FIXATION LOSSES: 0/13

FALSE POS ERRORS: $9 \%$

FALSE NEG ERRORS: $4 \%$

TEST DURATION: 03:28
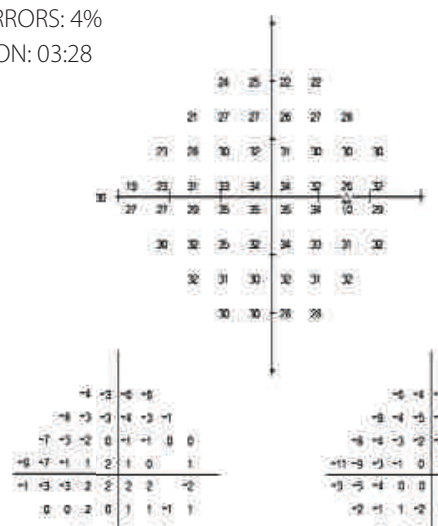

$0020,1+4$

$0-10-1$

- $a-2=$
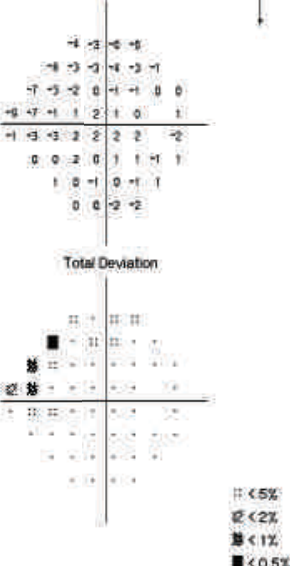

LEFT EYE

FIXATION LOSSES: $5 / 12 x x$

FALSE POS ERRORS: $18 \% \mathrm{xX}$

FALSE NEG ERRORS: $11 \%$

TEST DURATION: 03:45
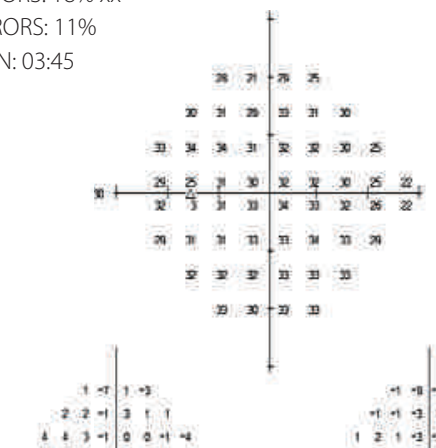

$4+3+100+1+4$

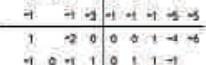

o. $101,1+$

1,1222

203

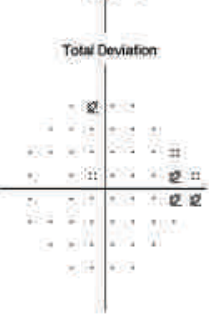

$0<5 \%$
$0<2 x$

$8<1 x$
$0<0.5 x$

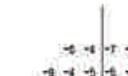

$-4-x+y-4=$

$x+4=2=2-2=$

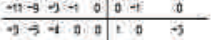

$2+1=0000$

$0+1=2+1=0$

$+2=4 \cdot 3$

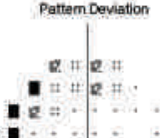

in.

-

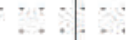

...

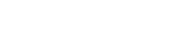

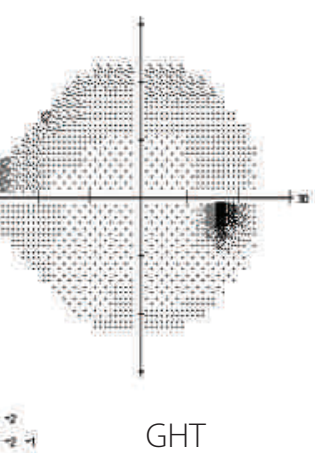

OUTSIDE NORMAL LIMITS

VFI 97\%

MD $-0.98 \mathrm{~dB}$

PSD $2.67 \mathrm{~dB} P<2 \%$
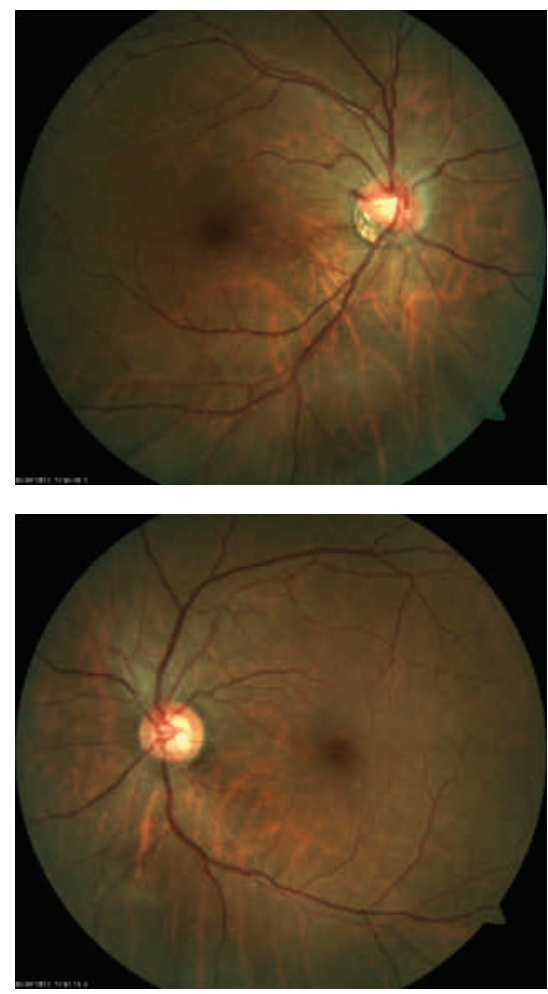

This case encapsulates the diagnostic dilemma of NTG.

The patient in question is a 51 -year old myopic (-7.00D) Asian female who discontinued treatment with prostaglandin analog two years ago. She takes no systemic medications, and denies any symptoms of systemic vascular dysregulation. Her IOPs are 14 and $15 \mathrm{mmHg}$; her CCTs are 494 and 493 microns.

The right $\mathrm{ONH}$ (top photo) is obliquely inserted, with superior temporal DH, inferior temporal BPPA, and adjacent RNFL defect. The left ONH shows inferior temporal NRR thinning with adjacent RNFL defect. Initial VF analysis (albeit with questionable reliability; confirmation pending) shows an early superior nasal step in both right and left. Her GP is being consulted to ensure that her systemic vascular status is satisfactory. Pending confirmatory VF analyses, topical treatment with prostaglandin analog (with a target pressure approaching the episcleral venous pressure of $\sim 10 \mathrm{mmHg}$ ) is likely to be initiated. 
31. Grise-Dulac A, et al. Assessment of corneal biomechanical properties in normal tension glaucoma and comparision with open-angle glaucoma, ocular hypertension, and normal eyes. J Glaucoma 2012;21:486-9.

32. Kaushik S, et al. Relationship between corneal biomechanical properties, central corneal thickness, and intraocular pressure across the spectrum of glaucoma. Am J Ophthalmol 2012;153: 840-9.

33. Ehrlich JR, et al. Goldmann applanation tonometry compared with corneal-compensated intraocular pressure in the evaluation of primary open-angle glaucoma. BMC Ophthalmol 2012 Sep 25;12(1):52 (epub ahead of print).

34. Ang GS, et al. Corneal biomechanical properties in primary open angle glaucoma and normal tension glaucoma. J Glaucoma 2008;17:259-62.

35. Flammer J, et al. The impact of ocular blood flow in glaucoma. Prog Ret Eye Research 2002;21:359-93.

36. Carter CJ, et al. Investigations into a vascular etiology for low-tension glaucoma. Ophthalmol 1990;97:49-55.

37. He Z, et al. The role of blood pressure in glaucoma. Clin Exp Optom 2011;94:133-49.

38. Moore D, et al. Dysfunctional regulation of ocular blood flow: a risk factor for glaucoma? Clin Ophthalmol 2008;4:849-61.

39. Drance SM, et al. Studies of factors involved in the production of low tension glaucoma. Arch Opbthalmol 1973;89:457-65.

40. Kaiser HJ, Flammer J. Systemic hypotension: a risk factor for glaucomatous damage? Ophthalmologica 1991;203:105-8.

41. Yanagi M, et al. Vascular risk factors in glaucoma: a review. Clin Experiment Ophthalmol 2011;39:252-8.

42. Deokule S, Weinreb RN. Relationships among systemic blood pressure, intraocular pressure, and openangle glaucoma. Can J Ophthalmol 2008;43:302-7.
43. Leske MC, et al. Risk factors for incident open-angle glaucoma. Ophthalmol 2008;115:85-93.

44. Caprioli J, Coleman AL. Blood pressure, perfusion pressure, and glaucoma. Am J Ophthalmol 2010;149:704-12.

45. Liu JHK, et al. Twenty-four-hour intraocular pressure pattern associated with early glaucomatous changes. Invest Ophthalmol Vis Sci 2003;44:1586-90.

46. Krasinska B, et al. A marked fall in nocturnal blood pressure is associated with the stage of primary openangle glaucoma in patients with arterial hypertension. Blood Pressure 2011;20:171-81.

47. Tokunaga T, et al. Association between nocturnal blood pressure reduction and progression of visual field defect in patients with primary open-angle glaucoma or normal-tension glaucoma. Jpn J Ophthalmol 2004;48:380-5.

48. Hulsman, CAA et al. Blood pressure, arterial stiffness, and open-angle glaucoma. Arch Ophthalmol 2007;125: 805-12.

49. De Moraes CG, et al. Risk factors for visual field progression in the Lowpressure Glaucoma Treatment Study. Am J Ophthalmol 2012;154:702-11.

50. Sung KR, et al. Twenty-four hour ocular perfusion pressure fluctuation and risk of normal-tension glaucoma progression. Invest Ophthalmol Vis Sci 2009;50:5266-74.

51. Sung KR, et al. Characteristics of visual field progression in medically treated normal-tension glaucoma patients with unstable ocular perfusion pressure. Invest Ophthalmol Vis Sci 2011;52:737-43.

52. Shoshani YZ, et al. Advances in the understanding of ocular blood flow in glaucoma. Expert Rev Ophthalmol 2010;5:189-200.

53. Plange N, et al. 24-h blood pressure monitoring in normal tension glaucoma: night-time blood pressure variability. J Human Hypertension 2006;20:137-42.

54. Drance SM. What can we learn from the disc appearance about the risk factors in glaucoma? Can J Opbthalmol 2008;43: 322-7.
55. Park HYL, et al. Nail bed hemorrhage. Arch Ophthalmol 2011;129:1299-1304.

56. Chung HS, et al. Peripapillary retinal blood flow in normal tension glaucoma. Br J Ophthalmol 1999;83: 466-9.

57. Emre M, et al. Ocular blood flow alteration in glaucoma is related to systemic vascular dysregulation. $\mathrm{BrJ}$ Ophthalmol 2004;88:662-6.

58. Jonas JB. Role of cerebrospinal fluid pressure in the pathogenesis of glaucoma. Acta Opbthalmol 2011;89: 505-14.

59. Pasquale LR. Low intracranial pressure: a tipping point in our understanding of primary open-angle glaucoma? Ophthalmol 2008;115:761-2.

60. Yang Y, et al. Role of cerebrospinal fluid in glaucoma: pressure and beyond. Medical Hypotheses 2012;74:31-4.

61. Ren R, et al. Cerebrospinal fluid pressure in glaucoma. Ophthalmol 2010;117:259-66.

62. Ren $\mathrm{R}$, et al. Cerebrospinal fluid pressure in ocular hypertension. Acta Ophthalmol 2011;89:142-8.

63. Berdahl JP, et al. Intracranial pressure in primary open angle glaucoma, normal tension glaucoma, and ocular hypertension: a case-control study. Invest Ophthalmol Vis Sci 2008;49:5412-8.

64. Berdahl JP, et al. Cerebrospinal fluid pressure is decreased in primary open-angle glaucoma. Ophthalmol 2008;115:763-8.

65. Wostyn $\mathrm{P}$, et al. Are intracranial pressure fluctuations important in glaucoma? Medical Hypotheses 2011;77:598-600.

66. Killer HE, et al. Cerebrospinal fluid exchange in the optic nerve in normaltension glaucoma. Br J Ophthalmol 2012;96:544-8.

67. Li Z, et al. Intraocular pressure vs intracranial pressure in disease conditions: a prospective cohort study (Beijing iCOP Study). BMC Neurology 2012;66:1-4.

68. Wang N, et al. Orbital cerebrospinal fluid space in glaucoma: the Beijing Intracranial Pressure (iCOP) Study. Ophthalmol 2012;119:2065-73. 
69. Caprioli J, Spaeth GL. Comparison of the optic nerve head in high- and low-tension glaucoma. Arch Opbthalmol 1985;103:1145-9.

70. Sugiyama K, et al. Disc haemorrhages in normal tension glaucoma. Asian J Ophthalmol 2000;2:6.

71. Cheung W, et al. Neuroprotection in glaucoma: drug-based approaches. Optom Vis Sci 2008;85:406-16.

72. Iester M, Mikelberg FS. Optic nerve head morphologic characteristics in high-tension and normaltension glaucoma. Arch Ophthalmol 1999;117:1010-3.

73. Tezel G, et al. Clinical factors associated with progression of glaucomatous optic disc damage in treated patients, Arch Ophthalmol 2001;119:813-8.

74. De Moraes CGV, et al. Spatially consistent, localized visual field loss before and after disc hemorrhage. Invest Ophthalmol Vis Sci 2009;50:4727-33.

75. Prata TS, et al. Factors affecting rates of visual field progression in glaucoma patients with optic disc hemorrhage. Ophthalmol 2010;117:24-9.

76. Drance SM. Disc hemorrhages in the glaucomas. Surv Ophthalmol 1989;33: 331-7.

77. Siegner SW, Netland PA. Optic disc hemorrhages and progression of glaucoma. Ophthalmol 1996;103:101424.

78. de Beaufort HC, et al. Recurrent disc hemorrhage does not increase the rate of visual field progression. Graefes Arch Clin Exp Ophthalmol 2010;248:839-44.

79. Susanna R, et al. Disc hemorrhages in patients with elevated intraocular pressure. Arch Ophthalmol 1979;97:2845.

80. Jonas JB, et al. Morphologic predictive factors for development of optic disc hemorrhages in glaucoma. Invest Ophthalmol V is Sci 2002;43:2956-61.

81. Nitta K, et al. Does the enlargement of retinal nerve fiber layer defects relate to disc hemorrhage or progressive visual field loss in normal-tension glaucoma? J Glaucoma 2011;20:189-95.

82. Radcliffe NM, et al. Anatomic relationship between disc hemorrhage and parapapillary atrophy. $A m \mathrm{~J}$

Ophthalmol 2008;146:735-40.

83. Healey PR, et al. Optic disc hemorrhages in a population with and without signs of glaucoma. Ophthalmol 1998;105:216-23.

84. Budenz DL, et al. Detection and prognostic significance of optic disc hemorrhages during the Ocular Hypertension Treatment Study. Ophthalmol 2006;113:2137-43.

85. Diehl DLC, et al. Prevalence and significance of optic disc hemorrhage in a longitudinal study of glaucoma. Arch Ophthalmol 1990;108:545-50.

86. Gloster J. Incidence of optic disc haemorrhages in chronic simple glaucoma and ocular hypertension. $\mathrm{BrJ}$ Ophthalmol 1981;65:452-6.

87. Yeung DYL, et al. Disc hemorrhage: what do we know? HKI Opbthalmol 2011;14:5-7.

88. Suh MH, Park KH. Period prevalence and incidence of optic disc haemorrhage in normal tension glaucoma and primary open-angle glaucoma. Clin Experiment Ophthalmol 2011;39:513-9.

89. Kim Y, et al. Risk factors associated with optic disc haemorrhage in patients with normal tension glaucoma. Eye 2010;24:567-72.

90. Drance SM, et al. The importance of disc hemorrhage in the prognosis of chronic open angle glaucoma. Arch Ophthalmol 1977;95:226-8.

91. Healy P. Optic disc haemorrhage: the more we look the more we find. Clin Experiment Opbthalmol 2011;39:485-6.

92. Collaborative Normal-Tension Glaucoma Study Group. The effectiveness of intraocular pressure reduction in the treatment of normaltension glaucoma. Am J Ophthalmol 1998;126:498-505.

93. Rasker MTE, et al. Rate of visual field loss in progressive glaucoma. Arch Ophthalmol 2000;118:481-8.

94. Rasker MT, et al. Deterioration of visual fields in patients with glaucoma with and without optic disc hemorrhages. Arch Ophthalmol 1997;115:1257-62.
95. Jonas JB, Xu L. Optic disk hemorrhages in glaucoma. Am J Ophthalmol 1994;118:1-8.

96. De Moraes CGV, et al. Risk factors for visual field progression in treated glaucoma. Arch Ophthalmol 2011;129: 562-8.

97. Leske MC, et al. Factors for glaucoma progression and the effect of treatment. Arch Ophthalmol 2003;121:4856.

98. Ishida $\mathrm{K}$, et al. Disk hemorrhage is a significantly negative prognostic factor in normal-tension glaucoma. Am J Ophthalmol 2000;129:707-14.

99. Collaborative Normal-Tension Glaucoma Study Group. Natural history of normal-tension glaucoma. Ophthalmol 2001;108:247-53.

100. Guo Y, et al. Five-year follow-up of parapapillary atrophy: The Beijing Eye Study. PLoS ONE 2012;7:e32005.

101. Sung KR. Disc hemorrhage: is that a risk factor or sign of progression? J Glaucoma 2012;21:275-6.

102. Kim SH, Park KH. The relationship between recurrent optic disc hemorrhage and glaucoma progression. Ophthalmol 2006;113:598-602.

103. De Moraes CG, et al. Rate of visual field progression in eyes with optic disc hemorrhages in the Ocular Hypertension Treatment Study. Arch Ophthalmol 2012 Aug 13:1-6;doi:10.1001/ archophthalmol.2012.2324 (epub ahead of print).

104. Shields MB. Hemorrhage of the optic disk in normal-tension glaucoma. Am J Ophthalmol 2000;129:796-7.

105. Jonas JB, et al. Parapapillary chorioretinal atrophy in normal and glaucoma eyes. Invest Ophthalmol V is Sci 1989;30:919-26.

106. Park KH, et al. Correlation between peripapillary atrophy and optic nerve damage in normal-tension glaucoma. Ophthalmol 1996;103:1899-1906.

107. Teng CS, et al. B-zone parapapillary atrophy and the velocity of glaucoma progression. Ophthalmol 2010;117: 909-15. 
108. Park HYL, et al. Optic disc torsion direction predicts the location of glaucomatous damage in normaltension glaucoma in patients with myopia. Ophthalmol 2012;xx:xxx (article in press).

109. Jonas JB, et al. Parapapillary atrophy in the chronic open-angle glaucomas. Graefes Arch Clin Exp Ophthalmol 1999;237:793-9.

110. Uchida H, et al. Increasing peripapillary atrophy is associated with progressive glaucoma. Ophthalmol 1998;105:1541-5.

111. De Moraes CG, et al. Predictive factors within the optic nerve complex for glaucoma progression: disc hemorrhage and parapapillary atrophy. Asia-Pac J Ophthalmol 2012;1:105-12.

112. Jonas JB, Xu L. Parapapillary chorioretinal atrophy in normalpressure glaucoma. Am J Ophthalmol 1993;115:501-5.

113. See JLS, et al. Rates of neuroretinal rim and peripapillary atrophy area change. Ophthalmol 2009;116:840-7.

114. Ehrlich JR, Radcliffe NM. The role of clinical parapapillary atrophy evaluation in the diagnosis of open angle glaucoma. Clin Ophthalmol 2010;4:971-6.

115. Lee EJ, et al. ß-zone parapapillary atrophy and the rate of retinal nerve fiber layer thinning in glaucoma. Invest Ophthalmol Vis Sci 2011;52:4422-7.

116. Jonas JB, et al. Parapapillary chorioretinal atrophy in normal and glaucoma eyes. Invest Ophthalmol Vis Sci 1989;30:908-18.

117. Pan YZ, et al. The relationship between peripapillary atrophy and primary open angle glaucoma. Asian J Ophthalmol 2008;10:114-7.

118. Collaborative Normal-Tension Glaucoma Study Group. Comparison of glaucomatous progression between untreated patients with normal-tension glaucoma and patients with therapeutically reduced intraocular pressures. Am J Ophthalmol 1998;126:487-97.

119. Caprioli J, Coleman AL. Intraocular pressure fluctuation. Ophthalmol 2008;115:1123-9.
120. Sommer A, et al. Clinically detectable nerve fiber atrophy precedes the onset of glaucomatous field loss. Arch Ophthalmol 1991;109:77-83.

121. Sung KR, et al. Retinal nerve fiber layer normative classification by optical coherence tomography for prediction of future visual field loss. Invest Ophthalmol Vis Sci 2011;52:2634-9.

122. Makabe K, et al. Longitudinal relationship between retinal nerve fiber layer thickness parameters assessed by scanning laser polarimetry (GDxVCC) and visual field in glaucoma. Graefes Arch Clin Exp Ophthalmol 2012;250:57581.

123. Leite MT, et al. Structure-function relationships using the Cirrus spectral domain optical coherence tomograph and standard automated perimetry. $J$ Glaucoma 2012;21:49-54.

124. Wollstein $G$, et al. Retinal nerve fibre layer and visual function loss in glaucoma: the tipping point. $\mathrm{BrJ}$ Ophthalmol 2012;96:47-52.

125. Ajtony C, et al. Relationship between visual field sensitivity and retinal nerve fiber layer thickness as measured by optical coherence tomography. Invest Ophthalmol Vis Sci 2007;48:258-63.

126. Wheat JL, et al. Correlating RNFL thickness by OCT with perimetric sensitivity in glaucoma patients. J Glancoma 2012;21:95-101.

127. Medeiros F, et al. A combined index of structure and function for staging glaucomatous damage. Arch Ophthalmol 2012;130:1107-16.

128. Greenfield DS. Visual field and intraocular pressure asymmetry in the Low-Pressure Glaucoma Treatment Study. Ophthalmol 2007;114:460-5.

129. Heijl A, et al. Reduction of intraocular pressure and glaucoma progression. Arch Ophthalmol 2002;120:1268-79.

130. Leske MC, et al. Predictors of long-term progression in the Early Manifest Glaucoma Trial. Ophthalmol 2007;114:1965-72.

131. Janz NK, et al. The Collaborative Initial Glaucoma Treatment Study. Opbthalmol 2001;108:1954-65.
132. Pasquale LR. Optimizing therapy for newly diagnosed open-angle glaucoma. Arch Ophthalmol 2008;126:125-7.

133. Lichter PR, et al. The investigators' perspective on the Collaborative Initial Glaucoma Treatment Study (CIGTS). Arch Ophthalmol 2008;126:122-4.

134. Danias J, Podos SM. Correspondence. Am J Ophthalmol 1999;127:623-6.

135. Goldberg I. Relationship between intraocular pressure and preservation of visual field in glaucoma. Surv Ophthalmol 2003;48[suppl 1];S3-7.

136. Cheng JW, et al. Meta-analysis of medical intervention for normal tension glaucoma. Ophthalmol 2009;116:1243-9.

137. Zeitz O, et al. Effects of glaucoma drugs on ocular hemodynamics in normal tension glaucoma: a randomized trial comparing bimatoprost and latanoprost with dorzolamide. BMC Ophthalmol 2005;5:11.

138. Natsis K, et al. Aerobic exercise and intraocular pressure in normotensive and glaucoma patients. BMC Ophthalmol 2009;9:6.

139. Weinreb RN, Levin LL. Is neuroprotection a viable therapy for glaucoma? Arch Ophthalmol 1999;117:1540-4.

140. Chang EE, et al. Glaucoma 2.0: neuroprotection, neuroregeneration, neuroenhancement. Ophthalmol 2012;119:979-86.

141. Gupta N, Yucel YH. Should we treat the brain in glaucoma? Can J Ophthalmol 2007;42:409-13.

142. Cordeiro MF, Levinn LA. Clinical evidence for neuroprotection in glaucoma. Am J Ophthalmol 2011;152:715-6.

143. Beidoe G, Mousa SA. Current primary open-angle glaucoma treatment and future directions. Clin Ophthalmol 2012;6:1699-1707. 